\title{
Advances in the understanding of multiferroics through soft $\mathrm{X}$-ray diffraction
}

T.A.W. Beale ${ }^{1}$, S.B. Wilkins ${ }^{2}$, R.D. Johnson ${ }^{3,4}$, D. Prabhakaran ${ }^{3}$, A.T. Boothroyd ${ }^{3}$, P. Steadman ${ }^{5}$, S.S. Dhesi ${ }^{5}$, and P.D. Hatton ${ }^{1, a}$

1 Department of Physics, University of Durham, South Road, Durham, DH1 3LE, UK

2 Department of Condensed Matter Physics \& Materials Science, Brookhaven National Laboratory, Upton, New York, 11973-5000, USA

3 Clarendon Laboratory, Department of Physics, University of Oxford, Parks Road, Oxford OX1 3PU, UK

4 ISIS Facility, Rutherford Appleton Laboratory - STFC, Chilton, Didcot, Oxfordshire OX11 0QX, UK

${ }^{5}$ Diamond Light Source Ltd., Harwell Science and Innovation Campus, Chilton, Didcot, Oxfordshire OX11 0DE, UK

Received 5 October 2011 / Received in final form 23 March 2012

Published online 15 June 2012

\begin{abstract}
The magneto-electric multiferroic $\mathrm{TbMn}_{2} \mathrm{O}_{5}$ has a complex magnetic structure in three different magnetically ordered phases. We have determined the nature of the induced magnetic order on the oxygen sites in the commensurate magnetic phase through full linear $\mathrm{X}$-ray polarisation analysis at the oxygen $K$ edge. This has been achieved rotating the linear polarisation of the incident beam at the source, and using multilayers to analyse the polarisation state of the scattered X-ray beam. We have confirmed that the anisotropy of the magnetic scattering at the oxygen edge is consistent with the anisotropy of the manganese magnetic structure.
\end{abstract}

\section{Introduction}

The spontaneous combination of multiple order parameters in a single system challenges our understanding of electron interactions. However the urge to comprehend such multiferroic materials is focussed by strongly coupled systems, whereby one ferroic order parameter can be controlled by a seemingly unrelated field (Fig. 1). Multiferroic materials have traditionally involved the coexistence of magnetic, ferroelectric and ferro-elastic order parameters [1]. It is logical to extend this to include more exotic order parameters such as electric quadrupole and magnetic torroidal order, with potential coupling between any of these. Transition metal oxides are seen as promising systems for displaying coupling between multiple order parameters as the energy of the order parameters tend to be of similar magnitudes.

The huge variety of potential uses for multiferroic materials is emphasised by the extensive use of piezo-electrics and piezo-magnetics, whereby the magnetic moments

\footnotetext{
${ }^{a}$ e-mail: p.d.hatton@dur.ac.uk
} 


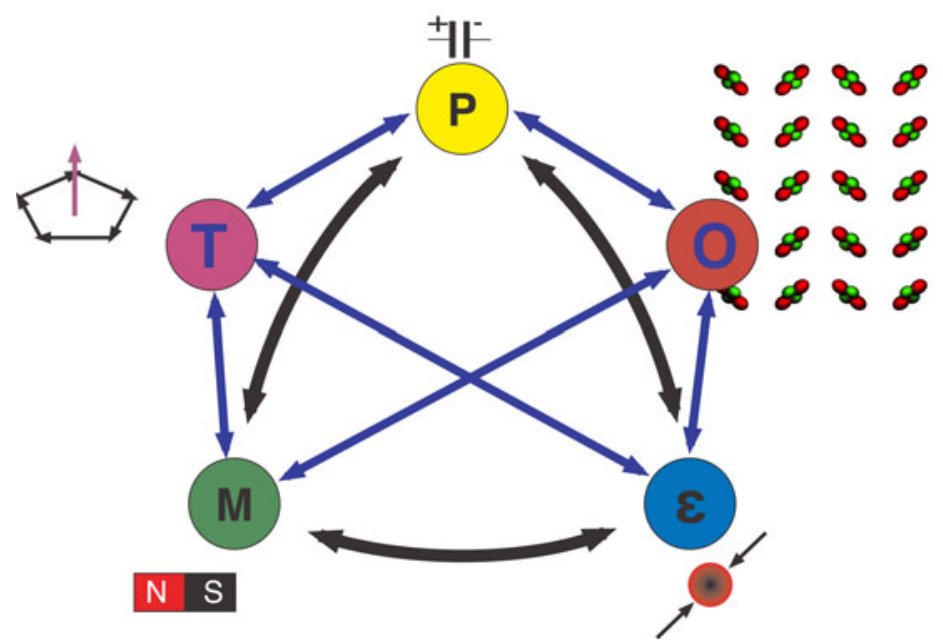

Fig. 1. Ferroelectric $(\mathrm{P})$, ferromagnetic $(\mathrm{M})$, and ferroelastic $(\varepsilon)$, are normally associated with multiferroic materials. Torroidal or anapole $(\mathrm{T})$, and orbital order $(\mathrm{O})$ form could extend multiferroic systems. In principle any of these five order parameters can couple to form a multiferroic system [1].

and ferroelectric dipoles can be controlled by a stress field and vice-versa. Similarly, magneto-electric multiferroics have inspired much research through the coupling of the magnetic and ferroelectric moments, either being driven by the opposing field. To date, such research has been unfruitful in commercial applications, principally due to (with the exception of $\mathrm{BiFeO}_{3}$ ), the low temperatures required.

Multiferroic materials, where multiple (anti)ferroic order parameters occur simultaneously have however been a subject of renewed interest [2]. This is in part driven by magneto-electric multiferroics, where there exists a strong coupling between the magnetic structure and the electric polarisation. This allows an electric field to control the magnetic structure in principle allowing the creation of hybrid magneto-electric devices.

Magneto-electric multiferroic materials can be divided into two categories, so called 'proper' and 'improper' ferroelectrics [2]. Proper multiferroics are usually defined as those where a structural transition breaks the inversion symmetry. Typically (for example $\mathrm{BiFeO}_{3}$ ), a central transition metal ion is surrounded by six oxygen ions forming an octahedra. The ferroelectric transition is then caused by a movement of the transition metal ion away from the centrosymmetric site at the centre of the octahedra. The ferroelectric dipole in 'improper' magneto-electic multiferroics is induced through a secondary order parameter that breaks inversion symmetry, often indirectly through movement of specific ions. This order parameter is often a magnetic order $[3,4]$, but can also be charge order [5-7], or potentially quadrupole (orbital) order.

The exact mechanisms causing the ferroelectric dipoles in different systems are not fully understood, however it is clear that both the magnetic and crystallographic symmetry are of utmost importance $[8,9]$. Inversion symmetry breaking ionic displacements are so minute that they cannot be observed through standard crystallographic determinations. As such a recent study determining these displacements through unconventional analysis [10] has sparked considerable interest, however it is uncertain whether such techniques can be developed for a range of systems or if this study was enabled through uniquely fortunate conditions. 
X-ray diffraction has proved an important tool in investigating the complex magnetic structures of multiferroic materials. Although neutron diffraction is still the most powerful technique for determining an overall magnetic structure [11-15], X-ray diffraction provides the ability to probe not only individual elemental contributions, but furthermore the hybridisation between different bands. Such hybridisation has been shown to polarise the $\mathrm{Tb} 5 d$ states by the manganese $4 p$ states in $\mathrm{TbMnO}_{3}$ [16], and a similar signal from hybridisation was also observed in $\mathrm{RMn}_{2} \mathrm{O}_{5}$ compounds [1719]. Of course the interest in these magneto-electric multiferroic materials is through the coupling between the ferroelectric polarisation and the magnetic structure. To date, and most likely due to experimental complexities, there have been relatively few X-ray studies where the effect of an electric field has been observed. Such electric field studies have however shown promise. Fabrizi et al. [20] used X-ray topography to observe the evolution of magnetic domains in the multiferroic $\mathrm{Ni}_{3} \mathrm{~V}_{2} \mathrm{O}_{8}$, while soft X-ray studies by Bodenthin et al. [21] and de Souza et al. [22] showed that the magnetic structure can be manipulated, and in the latter paper the helical component of the magnetic structure was reversed under an applied electric field.

The magneto-electric material $\mathrm{TbMn}_{2} \mathrm{O}_{5}$, is particularly interesting as the coupling between the magnetism and the electric polarisation is extremely strong [4], and although the low temperature of the multiferroic phase limits the viability of device construction, it provides an ideal platform for understanding the physics behind the interaction of the coupled order parameters. The magnetic structure of $\mathrm{TbMn}_{2} \mathrm{O}_{5}$ was determined initially through neutron diffraction [23]. Resonant X-ray diffraction was able to refine the direction of the magnetic order on the terbium ions in a band specific experiment [19]. We report full linear polarisation measurements $[19,24]$ at soft X-ray wavelengths from $\mathrm{TbMn}_{2} \mathrm{O}_{5}$. Using this technique we have measured the anisotropy of the antiferromagnetic moments on the manganese and oxygen ions in the magnetoelectric $\mathrm{TbMn}_{2} \mathrm{O}_{5}$. A signal, suggested to be magnetic, on the oxygen ions has been observed recently not only in $\mathrm{TbMn}_{2} \mathrm{O}_{5}$ [25], but also in $\mathrm{YMn}_{2} \mathrm{O}_{5}[22,26]$. These initial measurements have shown that the oxygen and transition metal oxide magnetic order parameter show similar temperatures however so far the measurements on the anisotropy have been limited to azimuthal measurements with large experimental errors. As the anisotropy of the signal gives a strong indication of the scattering tensor [27], we have sought to increase the accuracy of the scattering anisotropy. This has led us to conduct full linear polarisation analysis using soft X-rays, showing conclusively that the scattering anisotropy of the signal at the oxygen edge is determined by the manganese magnetic structure.

\section{Experimental details}

Single crystals of $\mathrm{TbMn}_{2} \mathrm{O}_{5}$ were obtained from the University of Oxford. $\mathrm{TbMn}_{2} \mathrm{O}_{5}$ crystallises into the orthorhombic Pbam spacegroup (Fig. 2) with lattice parameters $a=7.3251 \AA, b=8.5168 \AA$ and $c=5.6750 \AA$ [12]. Between $24 \mathrm{~K}$ and $38 \mathrm{~K}$ the magnetic structure is commensurate antiferromagnetic, with a wavevector of $(0.5,0,0.25)$, and is simultaneously ferroelectric. Above and below this phase the magnetic structure is incommensurate, and is yet to be fully determined.

Soft X-ray diffraction measurements were taken using the RASOR diffractometer [28] on the branch-line of the I06 beamline at Diamond Light Source. The linear polarisation state of the incident beam was rotated through $90^{\circ}$ using a single APPLE II undulator. The sample of $\mathrm{TbMn}_{2} \mathrm{O}_{5}$ was mounted upon a liquid helium flow cryostat providing a temperature range of $12-300 \mathrm{~K}$.

Full linear polarisation analysis has been developed in recent years as an alternative to azimuthal measurements (where the sample is rotated around the scattering 


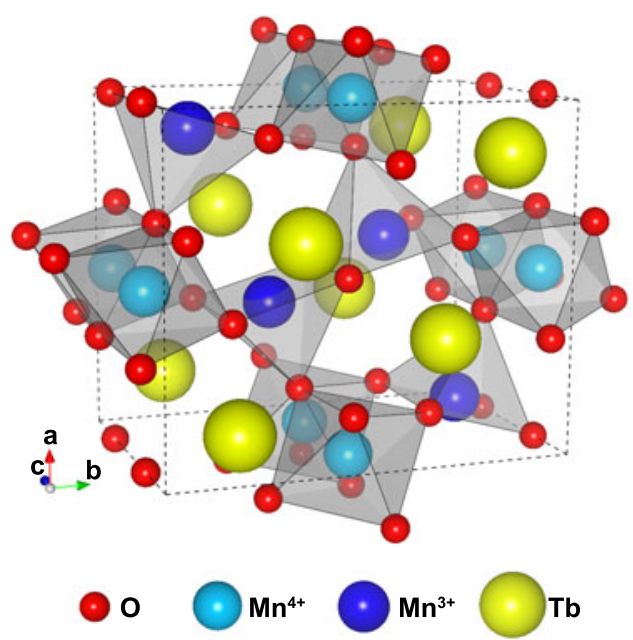

Fig. 2. The crystal structure of $\mathrm{TbMn}_{2} \mathrm{O}_{5}$. Note that the $\mathrm{Mn}^{3+}$ and $\mathrm{Mn}^{4+}$ ions are situated on different sites, the $\mathrm{Mn}^{4+}$ in the middle of $\mathrm{MnO}_{6}$ octahedra, and the $\mathrm{Mn}^{3+}$ just above the basal plane of a $\mathrm{MnO}_{4}$ square based pyramid.

vector). The technique instead rotates the incident $\mathrm{X}$-ray polarisation vector while measuring the polarisation state of the scattered beam, keeping the sample and detector stationary. This has the advantage of excluding intensity and polarisation variation through procession of the incident beam spot on the sample. In addition it removes the requirement for a sample rotation axis $(\phi)$, which is particularly useful for invacuum soft X-ray diffractometers. It should be noted that an azimuthal dependence and full linear polarisation analysis are not equivalent, indeed the azimuthal angle at which the full linear polarisation analysis is measured needs to be taken into account. However, both techniques take a (different) measurement of the anisotropy of the scattered signal.

In this experiment, the polarisation analysis of the scattered beam was conducted through secondary scattering from uniform $\mathrm{W} / \mathrm{B}_{4} \mathrm{C}$ multilayer samples (in contrast to a graded multilayer used by Staub et al. [31]). Analysis of the efficiency of the multilayer analysers using the main beam showed that the reflectivity of the multilayers on the Bragg condition was greater than $4 \%$ (oxygen) and $2.5 \%$ (manganese). As the multilayers are designed with periodicities matching the required energies, the $2 \theta$ angles were very close to $90^{\circ}$, and the leakthrough of the multilayers was less than $0.5 \%$.

\section{Results and discussion}

Figure 3 shows energy scans at the fixed wavevector $\boldsymbol{Q}=(0.5,0,0.25)$ corresponding to the commensurate magnetic reflection. The scan at the oxygen edge is comparable to data from a previous paper [25], however the increased intensity due to a higher incident flux allowed the reflection to be measured in a single scan. In addition the higher energy features in the resonance at $530.8 \mathrm{eV}$ and $534 \mathrm{eV}$ attributed to mixing of the unoccupied $t_{2 g}$ and $e_{g}$ spin bands with the oxygen $2 p$ states are much clearer. The lower panel of Fig. 3 shows the resonance at the manganese edge. In this resonance there are multiple pre-edge features below the main $L_{3}$ resonance at $644 \mathrm{eV}$. This resonant lineshape is somewhat different to that reported by Koo et al. [29], who show 

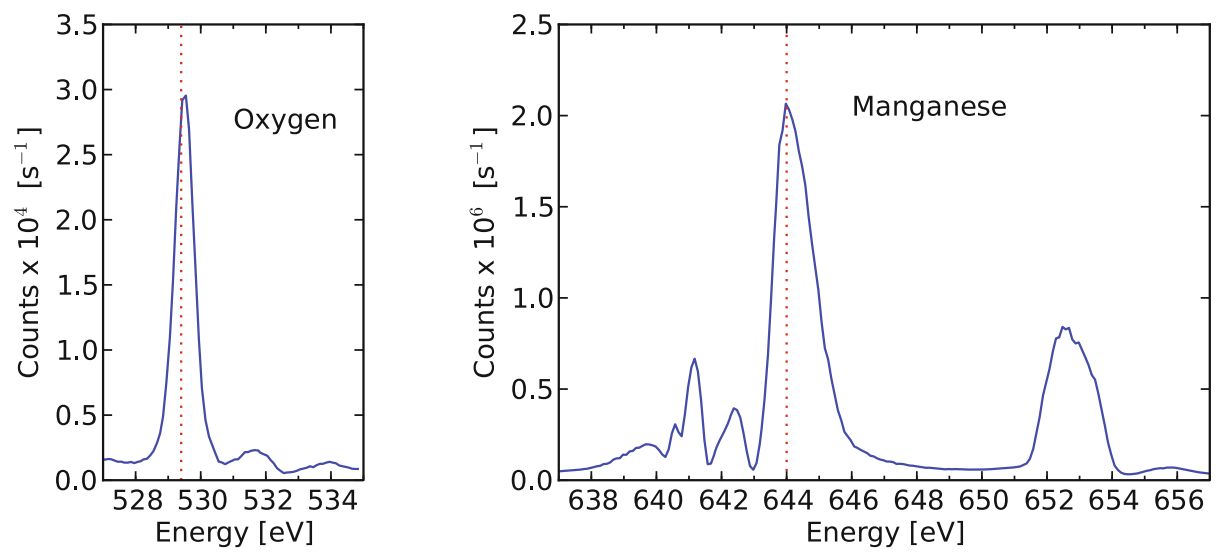

Fig. 3. Energy scans at fixed wavevector $\boldsymbol{Q}=(0.5,0,0.5)$ at the oxygen $K$ and manganese $L$ edges. The scans were taken with horizontal $(\sigma)$ incident polarised light, and a direct detector (no polarisation analysis). The vertical dotted lines show the energy at which the full polarisation data was collected. Scans are presented as measured, no corrections for self absorption have been made.
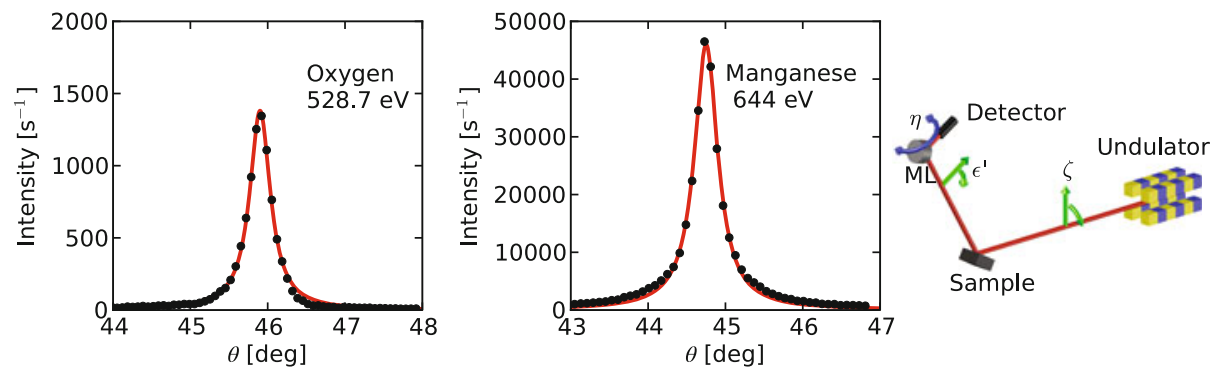

Fig. 4. The $(0.5,0,0.25)$ magnetic signal measured using multilayer analyser crystals at the oxygen and manganese edge. The scans were performed by a rocking the multilayer analyser through the Bragg reflection. The schematic on the right shows with experimental setup with the undulator rotating the incident polarisation $(\zeta)$ and the rotation $(\eta)$ of the multilayer (ML) and detector assembly to determine the Stokes parameters of the scattered polarisation $\left(\epsilon^{\prime}\right)$.

a similar intensity at the $L_{2}$ and $L_{3}$ edges, whereas we measure a $L_{3}$ edge intensity far greater than the intensity of the $L_{2}$. The resonance we show here is much more similar to that seen by Staub et al. [30] in $\operatorname{ErMn}_{2} \mathrm{O}_{7}$ leading us to speculate that the sample studied by Koo may have had a substantial magnetically 'dead' layer that affected their resonance. The measurements in this letter were taken at $28 \mathrm{~K}$ within the commensurate region. In addition, incommensurate reflections were observed above and below the commensurate phase at the manganese edge.

Figure 4 shows the diffraction of the magnetic $(0.5,0,0.25)$ reflection at the maximum signal of the resonances from the multilayer analyser crystals giving an efficiency of the multilayers of approximately $4 \%$ and $2.5 \%$ at the oxygen and manganese edges respectively, with horizontally $(\sigma)$ polarised incident light, and with $\eta=90$ such that the $\pi$ (rotated) channel of the magnetic scattering reflection is measured. Full linear polarisation analysis was conducted through the measurement of the exit beam polarisation as the incident beam polarisation $(\zeta)$ was rotated. Limitations of the undulator at I06 restricted the range of $\zeta$ to $0-90^{\circ}$, however the exit beam polarisation 


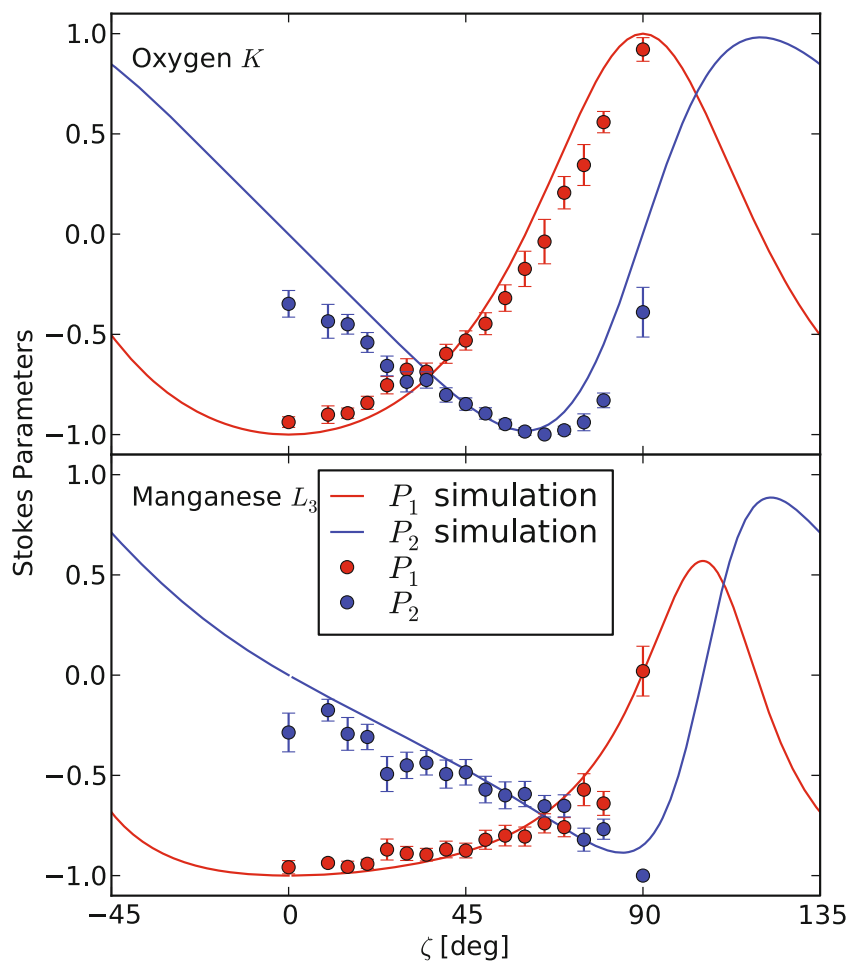

Fig. 5. Stokes parameters $P_{1}$ and $P_{2}$ measured at the oxygen $K$ edge and manganese $L_{3}$ edge as shown in the dashed lines in Fig. 3. The solid lines are simulations from the magnetic structure determined by Blake [12].

was measured through a rotation of $\eta$ of $180^{\circ}$. Integrated intensity of the rocking curve of the analyser was performed every $22.5^{\circ}$ of $\eta$ with the intensity fitted to a sinusoidal dependence. The Poincaré-Stokes parameters, $P_{1}$ and $P_{2}$, defined as

$$
P_{1}=\frac{I_{\sigma}-I_{\pi}}{I_{\sigma}+I_{\pi}}, \quad P_{2}=\frac{\left(I_{45^{\circ}}-I_{-45^{\circ}}\right)}{\left(I_{45^{\circ}}+I_{-45^{\circ}}\right)}
$$

where $I_{\sigma(\pi)}$ is in intensity of the reflection in the $\sigma$ and $\pi$ channel, and $I_{(-) 45^{\circ}}$ is the intensity of the light with a polarization vector rotated by $(-) 45^{\circ}$ from $\sigma$, are a measure of the angle and proportion of linear polarised light in a beam. $P_{1}$ and $P_{2}$ where calculated from the sinusoidal dependence of the integrated intensity with respect to $\eta$.

Figure 5 shows the dependence of $P_{1}$ and $P_{2}$ as a function of the incident beam polarisation $\zeta$. The solid lines are simulations of the Poincaré-Stokes parameters, using the magnetic and crystal structures previously published [12]. The simulation at the oxygen edge was calculated using the FDMNES code [32] using identical parameters as used previously to simulate the resonance [25]. The simulation at the manganese edge was performed by constructing the anisotropic scattering tensor as outlined by Johnson et al. [19]. The agreement between the simulations (where there are no floating parameters for optimising the fit), and the experiment is astonishing good. There is a slight deviation between the oxygen data and simulation, and we attribute this to the induced moment on the oxygen ions deviating slightly from the direction of the moment on the manganese. By comparison to the azimuthal measurement shown by Beale et al. [25], the data from the full linear polarisation 
analysis shown here has lower experimental error. This is principle enables a more sensitive analysis of the anisotropy of the magnetic scattering. In this case, it appears that the magnetic structure simulated with the azimuthal data [25] determined by neutron scattering [12] remains valid, and accurately describes the data presented here.

The results complement the anisotropic analysis by Johnson et al. [19] of the indirect measurement on the manganese magnetisation. In their publication they measured the induced moment in the terbium $5 d$ states, interpreting this as due to hybridisation with the manganese [12]. The results published here are a direct measurement of the antiferromagnetic moment on the magnetically active $3 d$ states, and the induced moment on the oxygen. By simulating the data with a model based on the magnetic moment directions determined by Blake [12], we are able to successfully explain our data. This establishes that the localised $3 d$ magnetic moment induces a moment on the hybridised oxygen ions. The observation of an induced moment on the oxygen ions in $\mathrm{TbMn}_{2} \mathrm{O}_{5}$ has been complemented by a similar measurement on $\mathrm{YMn}_{2} \mathrm{O}_{5}[22,26]$. In $\mathrm{YMn}_{2} \mathrm{O}_{5}$ the intensity of the oxygen resonance is observed to track the magnitude of the electric polarisation at not the magnitude of the magnet signal from the manganese edge. Such a result suggests that in both $\mathrm{YMn}_{2} \mathrm{O}_{5}$ and $\mathrm{TbMn}_{2} \mathrm{O}_{5}$ the signal and the oxygen edge is directly related to the magneto-electric mechanism. In fact Partzsch et al. [26] claim that the electronic contribution [33,34] plays a major role in the ferroelectric mechanism in these materials.

\section{Conclusion}

The X-ray diffraction signal observed at the oxygen $K$ absorption edge in $\mathrm{TbMn}_{2} \mathrm{O}_{5}$ has changed our understanding of the hybridisation in multiferroic materials. In this paper we have shown data that demonstrates that the origin of the reflection is the hybridisation between the oxygen and manganese ions. This has been shown through an accurate measurement of the anisotropy of the oxygen signal. This measurement has demonstrating the potential of full linear polarisation analysis with soft X-ray diffraction enabling future measurements directly probing not only magnetic structures, but also anisotropic orbital order. The work at Brookhaven National Laboratory is supported by the Office of Science, U.S. Department of Energy, Division of Materials Science, under contract no. DE-AC02-98CH10886.

\section{References}

1. N.A. Spalding, S.-W. Cheong, R. Ramesh, Phys. Today 63, 38 (2010)

2. S.-W. Cheong, M. Mostovoy, Nature Materials 6, 13 (2007)

3. M. Kenzelamnn, A.B. Harris, S. Jonas, C. Broholm, J. Schefer, S.B. Kim, C.L. Zhang, S.-W. Cheong, O.P. Vajk, J.W. Lynn, Phys. Rev. Lett. 95, 087206 (2005)

4. N. Hur, S. Park, P.A. Sharma, J.S. Ahn, S. Guha, S.-W. Cheong, Nature 429, 392 (2004)

5. N. Ikeda, H. Ohsumi, K. Ohwafa, K. Ishii, T. Inami, K. Kakurai, Y. Murakami, K. Yoshii, S. Mori, Y. Horibe, H. Kitô, Nature 436, 1136 (2005)

6. G.T. Rado, J.M. Ferrari, Phys. Rev. B 12, 5166 (1975)

7. G.T. Rado, J.M. Ferrari, Phys. Rev. B 15, 290 (1977)

8. P. Toledano, Phys. Rev. B 79, 094416 (2009)

9. P. Toledano, W. Schranz, G Krexner, Phys. Rev. B 79, 144103 (2009)

10. C. Azimonte, E. Granado, H. Terashita, S. Park, S.-W. Cheong, Phys. Rev. B 81, 012103 (2010)

11. M. Kenzelmann, A.B. Harris, S. Jonas, C. Broholm, J. Schefer, S.B. Kim, C.L. Zhang, S.-W. Cheong, O.P. Vajk, J.W. Lynn, Phys. Rev. Lett. 95, 087206 (2005) 
12. G.R. Blake, L.C. Chapon, P.G. Radaelli, S. Park, N.H. Hur, S.-W. Cheong, J. RodriguezCarvajal, Phys. Rev. B 71, 214402 (2005)

13. M. Fukunaga, K. Nishihata, H. Kimura, Y. Noda, K. Kohn, J. Phys. Soc. Jpn. 76, $074710(2007)$

14. B. Roessli, P. Fischer, P.J. Brown, M. Janoschek, D. Sheptyakov, S. Gvasaliya, B. Ouladdiaf, O. Zaharko, E. Golovenchits, V. Sanina, J. Phys. C 20, 485216 (2008)

15. P.G. Radaelli, C. Vecchini, L.C. Chapon, P.J. Brown, S. Park, S.-W. Cheong, Phys. Rev. B 79, 020404 (2009)

16. D. Mannix, D.F. McMorrow, R.A. Ewings, A.T. Boothroyd, D. Prabhakaran, Y. Joly, B. Janousova, C. Mazzoli, L. Paolasini, S.B. Wilkins, Phys. Rev. B 76, 184420 (2007)

17. R.A. Ewings, A.T. Boothroyd, D.F. McMorrow, D. Mannix, H.C. Walker, B.M.R. Wanklyn, Phys. Rev. B 77104415 (2008)

18. G. Beutier, A. Bombardi, C. Vecchini, P.G. Radaelli, S. Park, S.-W. Cheong, L.C. Chapon, Phys. Rev. B 77, 172408 (2008)

19. R.D. Johnson, S.R. Bland, C. Mazzoli, T.A.W. Beale, C.H. Du, C. Detlefs, S.B. Wilkins, P.D. Hatton, Phys. Rev. B 78, 104407 (2008)

20. F. Fabrizi, H.C. Walker, L. Paolasini, F. de Bergevin, T. Fennell, N. Rogado, R.J. Cava, Th. Wolf, M. Kenzelmann, D.F. McMorrow, Phys. Rev. B 82, 024434 (2010)

21. Y. Bodenthin, U. Staub, M. García-Fernández, M. Janoschek, J. Schlappa, E.I. Golovenchits, V.A. Sanina, S.G. Lushnikov, Phys. Rev. Lett. 100, 027201 (2008)

22. R.A. de Souza, U. Staub, V. Scagnoli, M. Garganourakis, Y. Bodenthin, S.-W. Huang, M. García-Fernández, S. Ji, S.-H. Lee, S. Park, S.-W. Cheong, Phys. Rev. B 84, 104416 (2011)

23. L.C. Chapon, G.R. Blake, M.J. Gutmann, S. Park, N. Hur, P.G. Radaelli, S.-W. Cheong, Phys. Rev. Lett. 93, 177402 (2004)

24. V. Scagnoli, C. Mazzoli, C. Detlefs, P. Bernard, A. Fondacaro, L. Paolasini, F. Fabrizi, F. de Bergevin, J. Sync. Rad. 16, 778 (2009)

25. T.A.W. Beale, S.B. Wilkins, R.D. Johnson, S.R. Bland, Y. Joly, T.R. Forrest, D.F. McMorrow, F. Yakhou, D. Prabhakaran, Phys. Rev. Lett. 105, 087203 (2010)

26. S. Partzsch, S.B. Wilkins, J.P. Hill, E. Schierle, E. Weschke, D. Souptel, B. Büchner, J. Geck, Phys. Rev. Lett. 107, 057201 (2011)

27. J.P. Hannon, G.T. Trammell, M. Blume, Doon Gibbs, Phys. Rev. Lett. 61, 1245 (1988)

28. T.A.W. Beale, T.P.A. Hase, T. Iida, K. Endo, P. Steadman, A.R. Marshall, S.S. Dhesi, G. van der Laan, P.D. Hatton, Rev. Sci. Instr. 81073904 (2010)

29. J. Koo, C. Song, S. Ji, J.-S. Lee, J. Park, T.-H. Jang, C.-H. Yang, J.-H. Park, Y.H. Jeong, K.-B. Lee, T.Y. Koo, Y.J. Park, J.-Y. Kim, D. Wemeille, A.I. Goldman, G. Srajer, S. Park, S.-W. Cheong, Phys. Rev. Lett. 99, 197601 (2007)

30. U. Staub, Y. Bodenthin, M. García-Fernández, R.A. de Souza, M. Garganourakis, E.I. Golovenchits, V.A. Sanina, S. G. Lushnikov, Phys. Rev. B 81, 144401 (2010)

31. U. Staub, V. Scagnoli, Y. Bodenthin, M. García-Fernández, R. Wetter, A.M. Mulders, H. Grimmer, M. Horisberger, J. Sync. Rad. 15, 469 (2008)

32. Y. Joly, Phys. Rev. B 63, 125120 (2001)

33. A.S. Moskvin, R.V. Pisarev, Phys. Rev. B 77, 060102 (2008)

34. G. Giovannetti, J. van den Brink, Phys. Rev. Lett. 100, 227603 (2008) 